\title{
"Mean fractal dimension" of blood ultrasonic Doppler signals
}

\author{
Jean-Marc Girault, Member, IEEE, Denis Kouamé, Member and F r édríc Patat
}

\begin{abstract}
A bstract- This paper deals with the characterization of blood ultrasonic Doppler signals via the computation of the "mean fractal dimension". We sho w and we discuss the existing links betw een the "mean fractal dimension" and the well-known mean frequency and the bandwidth usually used to characterize the blood Doppler signals. T ofnish, $w$ e calculate and ve discuss all these parameters in the case of in vivo blood Doppler signals.

Keywords - Fractal dimension, Length, Ultrasound Doppler signal .
\end{abstract}

\section{INTRODUCTION}

$\mathrm{T}$ $7 \mathrm{H}$ e main purpose that $\mathrm{w}$ ewish to give to this $\mathrm{w}$ ork is to study the contribution of the fractal dimension estimation to characterize narrow band stochastic signals like the blood ultrasonic Doppler signals.

The idea which underlies this work is based on the fact that the $1 / f^{\alpha}$ noises, characterized by fractal dimension, are obtained for example by low-pass filtering of a white noise. We think that the blood ultrasonic Doppler signal which can be obtained by filtering band pass of a white noise, can also be characterized by fractal dimension.

This work, which contin ues the idea of Wang [1] on the characterization of blood ultrasonic Doppler signals, by the computation of fractal dimension, will try to answer the questions left outstanding by the author.

More precisely, our study will consist in on the one hand justifying the computation of fractal dimension of a priori non fractal signals and on the other hand to clarify in details the similarity betw een the temporal evolution in the short time of fractal dimension and the mean frequency (or maximum) of the Doppler spectrum.

To achiev e our goal ve must answer the following question: can the fractal theory be applied within the framew ork of our study?

To answer it, it is initially necessary to point out what are the blood ultrasonic Doppler signals and fractal signals.

Another question is to kno wwhich are the parameters which make it possible indeed to characterize them.

We will initially give some brief replies with regard to the Doppler signals then in a second time the signals of fractal c haracter.

\section{THE BLOOHPLER SIGNAL}

Because of the random spatial distribution of red blood cells and the dynamics of the cardiovascular system, the Doppler signal is considered as a time-varying random signal. This signal which has a non-stationary character in

The authors are with the LUSSI/GIP ULtrasons, Tours, France. E-mail: jmgirault@univ-tours.fr. frequency can be considered stationary on a time interval of duration low er or equal to $10 \mathrm{~ms}$.

This signal can be modeled coarsely by a narrow band random signal modulated at the same time in amplitude and frequency:

$$
z(t)=\rho(t) e^{j \phi(t)}
$$

where $\rho(t)$ is the instantaneous magnitude of the analytic signal, indicating the amplitude modulation and $\phi(t)$ is the instantaneous phase specifying the Doppler frequency modulation. Indeed, both instantaneous energy and frequency of the Doppler signal are very dependent on the various phases on the cardiac cycle.

In the literature, it was shown that Doppler signals could be modeled by parametric models as for example AR (autoregressive) model. The modeled signals are nothing other than colored noises obtained by narrow band AR filters [2] or b y other ypes of filtering[3].

Let us recall that this aspect of filtering, is a key point since, as $\mathrm{w}$ ewill see it thereafter, the fractal signals can also be obtained by filtering.

\section{FRACTAL SIGNALS}

A signal can be described as fractal if it is con timous and non-derivable and if it presents:

- deterministic or statistical self-similarities ,

- deterministic or statistical self-affinities,

and this on all the scales and thus at all the frequencies.

Let us specify that there exists in the nature of the signals which present a fractal character only in one restricted range of frequency. Moreover, let us add that it is also the case in practice, a numerical signal will be fractal only on one range of limited scale: the smallest scale will be defined by the sampling period and thdargest $b$ y the size of the analyzed signal.

F urthermore, let us specify that if we do not have a priori kno wledge on the fractal baracter of a random signal, nothing enables us to affirm that it has or not fractal properties.

T ocharacterize such signals, there is a certain number of parameters. The simplest and the most commonly used is the fractal dimension $D$. The latter makes it possible to evaluate thdegree of regularit $y$ or self-affinity present in the signal. Let us note that a very irregular signal, will fill all space and fractal dimension is worth $D \approx 2$ whereas a regular signal rather has a value of fractal dimension near to the unity: $D \approx 1$.

Another aspedev eloped for better understanding the fractal signals is the implementation of mathematical mod- 
els, as for examples the fractional brownian motion [4] known as $1 / f^{\alpha}$ noises, where $\alpha=(5-D) / 2$ and where $D$ is fractal dimension. Let us note that a white noise is a $1 / f^{0}$ noise.

Such noises can be obtained for example starting from the fARIMA model [5] or by summation of several signals resulting by the filtering of a white noise through low-pass filters of different cut-off frequencies [6].

Here, as we will see it thereafter, it is rather the opposite operation which will interest us, in the sense where it is rather the filter or the system characteristics which we will try to evaluate starting from the filtered stochastic signal.

\section{MEASURE OF THE FRACTAL DIMENSION}

After having introduced the various models of fractal signals, let us clarify the technique chosen to evaluate fractal dimension such signals.

In the case of signals analysis (in opposite to the study of graphs or images), the technique which seems the best adapted is that of the length measure. It is Mandelbrot [7] with the help of the Richardson study [8] that showed the length could expressed according to fractal dimension $D$ by:

$$
L(k)=L_{0} k^{1-D},
$$

where $k$ is a gauge which plays the role of a zoom and where $L_{0}$ is a constant.

In the case of the study of numerical signals, Eq.(2) becomes:

$$
L(K)=T^{D} K^{1-D},
$$

where $K=k T_{s}$ is the experimental gauge, $T_{s}$ is the sampling period and $T$ is the duration of the window of analysis, duration which will be selected to ensure the stationarity of the studied signal. In our case this value will be normalized and will be worth the unity. On the formula above, one sees that fractal dimension can be simply obtained while calculating, by linear regression, the slope of the curve log-log of according to $K$.

After having introduced the link between the length and the fractal dimension, focus to a length estimator. Let us note that there exist several length estimators as for example the one proposed by [9]. Here we only present the simplest.

The presented estimator was introduced by Burlaga and Klein [10] to measure the length of signals resulting from the interplanetary magnetic fields. This estimator based on the " $\mathrm{L} 1$ norm" is expressed by:

$$
L_{B K}(n, k)=\frac{1}{k} \sum_{n=1}^{N-k}\left|z\left(n T_{s}\right)-z\left((n+k) T_{8}\right)\right|
$$

where $n$ is the current time, $k$ is called gauge, $N$ is the number of points of the studied signal.

This length estimator is a generalized length estimator, in the sense that instead of having one sampling period $T_{s}$ between two consecutive points (as it is the case for the Euclidean length), it is rather a multiple of the sampling period $k T_{s}$ with $k=1,2 \ldots k_{\max }$. This later point indicates

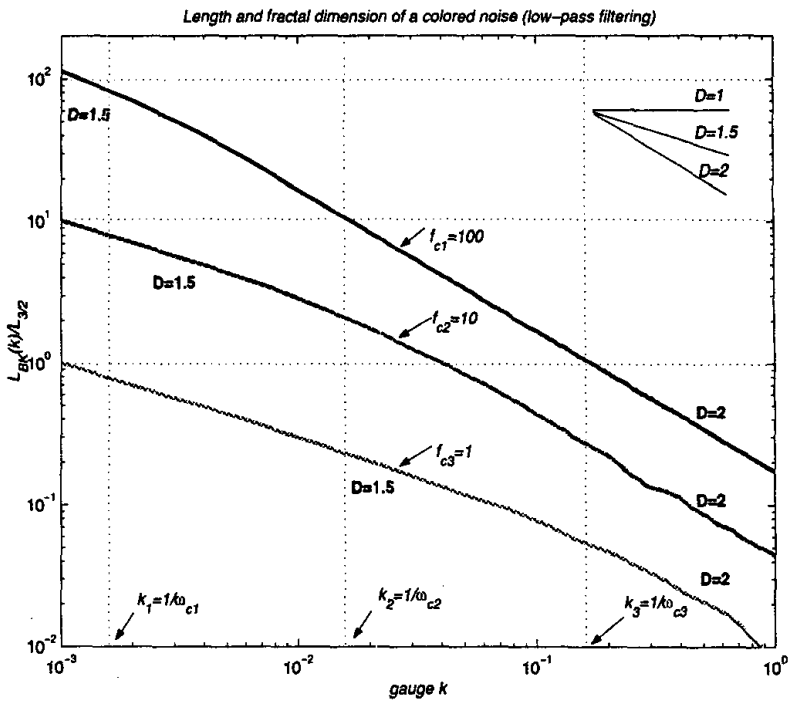

Fig. 1.

Length of colored noises obtained by a first order low-pass filter of the Lorentz type for various values of the cut-off frequency $\left(f_{c 1}=100, f_{c 2}=10\right.$ and $\left.f_{c 3}=1\right)$, the sampling frequency is $f_{s}=1000$.

that the estimated length is not the length of the studied signal but rather the length of a filtered signal since this one results from a decimation and an interpolation of firstorder.

\section{MEASURE OF THE LENGTH AND FRACTAL DIMENSION OF FILTERED RANDOM SIGNALS}

Having all tools necessary for our study, let us focus to the length, then to the fractal dimension in the case of colored signals by low-pass filtering, and in the second time in the case of colored signals by filtering band pass.

\section{A. Low-pass Filter}

Let us consider a simple first order low-pass filter (Lorentz type) defined by the square modulus of its transfer function:

$$
\left|H_{0}(\omega)\right|^{2}=\frac{1}{1+\left(\omega / \omega_{c}\right)^{2}}
$$

where $\omega_{c}$ is the cut-off frequency of the filter.

It was shown by [6] that the length of the noise thus filtered, which is named "a random walk" (or first-order Markov process), is proportional to the cut-off frequency in a restricted range of frequency.

As it is indicated in table (I), there are three jointed areas where fractal dimension (slope of the curve) take different values. Remark, as it is illustrated on the Fig. (1), that there are only two observed areas, the first one corresponds to $D=1.5$ and the second to $D=2$, the third could not be reach. It is clear in this case that the gauge values at 
TABLE I

\begin{tabular}{|c|c|c|c|}
\hline gauge & $k \gg \sqrt{\frac{\omega_{c}}{2}}$ & $\sqrt{\frac{\omega_{c}}{2}}>k>\frac{1}{\omega_{c}}$ & $k<\frac{1}{\omega_{c}}<\sqrt{\frac{\omega_{c}}{2}}$ \\
\hline$L_{B K}(k)$ & $L_{1} k^{1-D}$ & $L_{2} \sqrt{f_{c}} k^{1-D}$ & $L_{3 / 2} f_{c} k^{1-D}$ \\
\hline$D$ & 1 & 2 & 1.5 \\
\hline King of & continuous & white & random \\
signal & signal & noise & walk \\
\hline
\end{tabular}

Length and fractal dimension according to the various values of gauge $k ; L_{1}, L_{2}$ and $L_{3 / 2}$ are tree constants.

the jointed areas points $\left(k_{1}=1 / \omega_{1}, k_{2}=1 / \omega_{2}, k_{3}=1 / \omega_{3}\right)$ are also functions of the cut-off frequency.

If one calculates the average slope of the logarithm length according to the logarithm of the gauge, one sees that because of the various slopes, the associated fractal dimension corresponds to an average value of the fractal dimension.

\section{B. Band pass filter}

With an aim of simplicity, suppose that the narrow band is sufficiently weak to assimilate the filtered noise as a sinusoidal signal. The modulus of the transfer function of the band pass filter is written in this case, i.e. when $\omega_{0} / \omega_{c} \ll 1$ :

$$
|H(\omega)| \approx\left|H_{0}\left(\omega-\omega_{0}\right)\right|+\left|H_{0}\left(\omega+\omega_{0}\right)\right|
$$

where $\omega_{0}$ is the central frequency and $2 \omega_{c}$ is the bandwidth.

The length measure highlights several significant points illustrated on the Fig.(2):

- firstly when the gauge is a multiple of the period of the sinusoidal signal, the evaluated length presents local minima. Indeed let us suppose that the sinusoidal signal period, $T_{0}=1 / f_{0}$, that is observed over one duration equals to $10 T_{0}$, then if the gauge is worth $k=T_{0}$, the length is worth $10 T_{0}$, whereas if the gauge takes its maximum value $k=10 T_{0}$ the length is worth $10 T_{0}$ too;

- secondly when the gauge takes its minimum value, $k=$ $T_{s}$, the length takes its maximum value;

- thirdly, when the gauge takes a multiple value of the halfperiod $k=T_{0} / 2$ then the length presents local maxima which follow the same slope as that of a white noise. Be careful, starting from the graphs of the Fig.(2), one would not have to conclude too hastily only the Doppler signal has a fractal character. Indeed, although these successive points have a slope indicating a fractal dimension $D=2$, it seems that this belıavior results simply from the decimation and the periodicity of the Doppler signal.

If one measures signal fractal dimension on all the scale range, as it is the case in work of Wang [1], this one seems being dependent on the central frequency of the filter and its band-width. Here, in opposite to the Wang study, we show (see Figs.(2) and (3)) and demonstrate its dependence (see below). This fractal dimension does not correspond any more to the usual sense of the term but rather to an average fractal dimension.

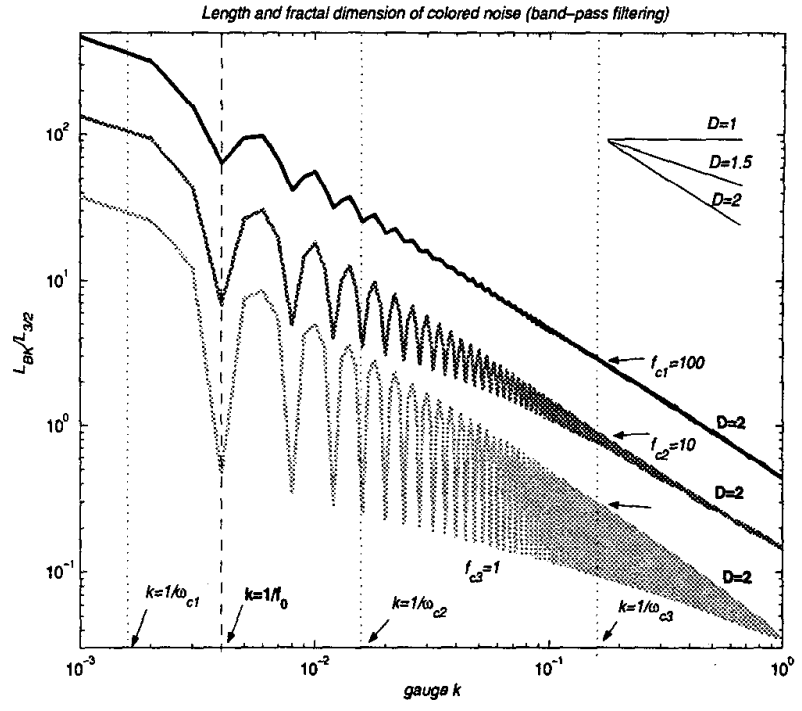

Fig. 2.

Length of filtered noises obtained by a band pass filter for a constant value of the central frequency $f_{0}=250$ and for different values of the cut-off frequency $f_{c 1}=100$, $f_{c 2}=10$ and $f_{c 3}=1$, the sampling frequency is $f_{s}=1000$.

In fact, one can show that the length of a narrow band signal is connected to the central frequency [11] and its bandwidth by the following relation:

$$
L_{B K}=\beta \sqrt{\kappa \omega_{c}^{2}+\omega_{0}^{2}}
$$

where $\kappa$ and $\beta$ are constants. Here the magnitude of the analyzed signal will be set to one. Note that in [11], it is proposed a new method to evaluate the mean frequency. Under these conditions the mean fractal dimension can be expressed as a function :

$$
D=1-g\left(\log \left(\omega_{c}^{2}+\omega_{0}^{2}\right)\right)
$$

where $g$ is a function related to the derivative of the logarithm of the gauge according to the logarithm of the gauge.

In short, one highlighted that the measured length is dependent on filtering used. In the case of low-pass filters, the dependence appears with the cut-off frequency whereas in the case of band pass filters the dependence appears at the same time with the cut-off frequencies but also with the central frequency.

\section{FRACTAL DIMENSION OF BLOOD ULTRASOUND DOPPLER SIGNALS}

Focus now to in vivo signals and let us evaluate the mean fractal dimension of such signals. We have illustrated on the Fig.(4) a typical encountered Doppler signal (carotid artery Doppler signal). As it has been already introduced, it can be observed, from the Fig.(4b), the time-varying behavior (frequency mon-stationarities) of the Doppler signal 


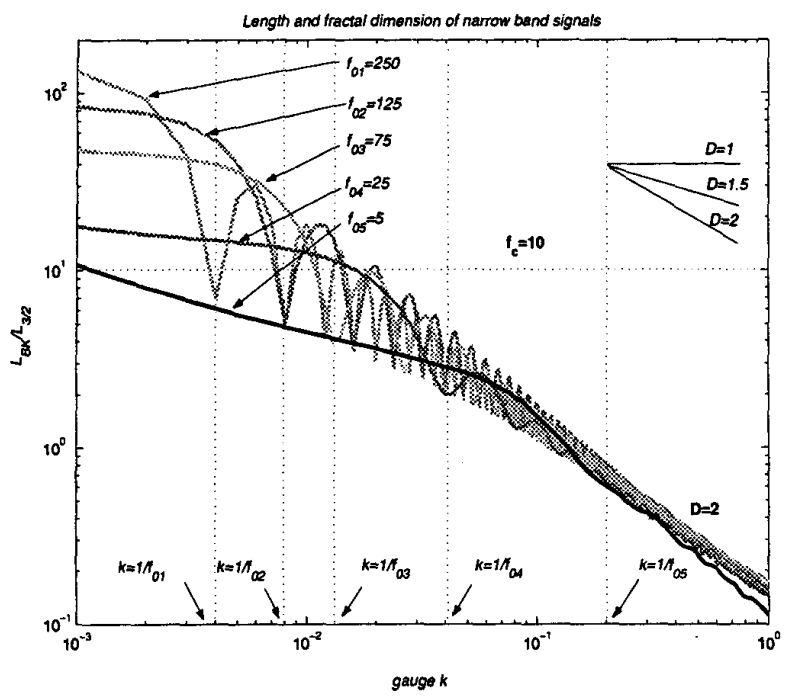

Fig. 3.

Length of filtered noises obtained by a band-pass filter for different values of the central frequency $f_{01}=250$,

$f_{02}=125, f_{03}=75, f_{04}=25, f_{05}=5$ and for a constant cut-off frequency $f_{c}=10$, the sampling frequency is

$$
f_{s}=1000 \text {. }
$$

at the different cardiovascular phases (the cardiac cycle is about 1 beat per second). From the time-frequency representation, we can extract frequency parameters to characterize for example of arterial stenosis; the most commonly used are the mean frequency and the bandwidth (see Fig.(4c)). On Fig.(4d) we show that the mean fractal dimension follows the cardiac cycle as expected. The latter fluctuates around values ranging between 1 and 2 what corresponds well to our waitings.

We think that the mean fractal dimension is an interesting parameter in the sense where it provides a synthetic parameter which describes at the same time its dependence according to the central frequency and of the band-width. However this advantage can be regarded as a disadvantage especially if we want to follow the evolution of only one of the two parameters.

\section{CONCLUSION}

In this study, after having recalled the theoretical aspects, we have in a first time demonstrated the link between the mean fractal dimension and the characteristic parameters of the filter and in a second step we have discussed and demonstrated why it is significant.

\section{REFERENCES}

[1] Y. Wang et W. Wang, "Fractal concept and its analysis method for doppler ultrasound signals," Ultrasound in Med. Biol., vol. 19 , pp. 661-666, 1993.

[2] J.M. Girault, D. Kouamé, A. Ouahabi, and F. Patat, "Estimation of the blood doppler frequency shift by a time-varying approach," Ultrasonics, vol. 38, pp. 682-687, 2000.

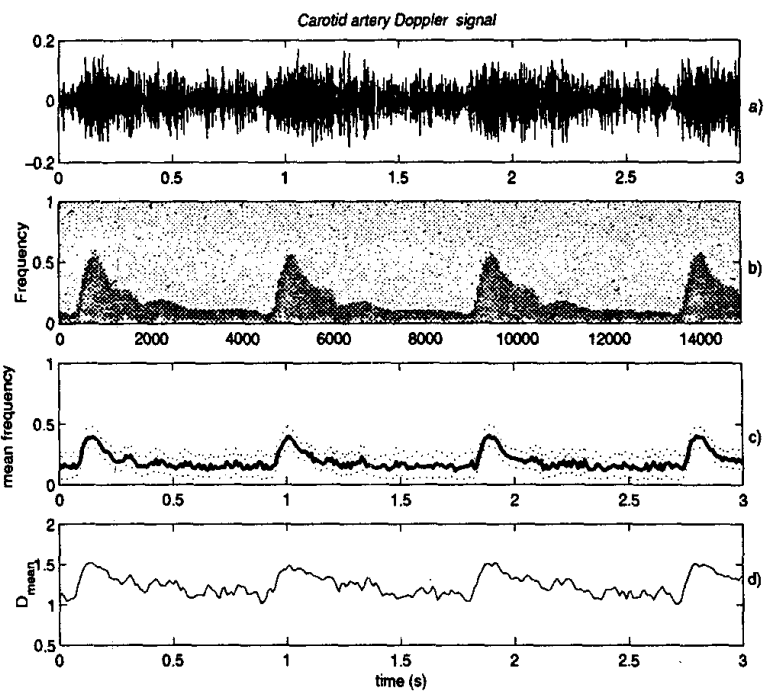

Fig. 4.

Fractal dimension of blood ultrasound Doppler signals from a carotid artery.a) Doppler signal, b) spectrogram of the Doppler signal, c) mean frequency $\left(\omega_{0}, \omega_{0}+/-\omega_{c} / 2\right.$ and d) mean fractal dimension.)

[3] B.A.J Angelsen and K. Kristoffersen, "Discrete time estimation of the mean doppler frequency in ultrasonic blood velocity measurements," EEE Trans. on Biomed. Eng., vol. 30, pp. 207-217, 1983.

[4] B.B. Mandelbrot and J.W. Van Ness, "Fractional brownian motions, fractional noises ans applications," SIAM Review, vol. 10, pp. 422-437, 1968.

[5] J.R.M. Hosking, "Fractional differencing," Biometrika, vol. 68 , pp. 165-176, 1981.

[6] J.J. Gagnepain, J. Groslambert, and R. Brendel, "The fractal dimension of phase and frequency noises : anaother approach to oscillator characterization," Proceeding of the Conf. on Metrol. ogy and Properties of Engineering Surfaces, Teeside Polytchnic, Middleborouh, England, pp. 113-118, 1985.

[7] B. Mandelbrot, The fractal geometry of nature, San Francisco: Freeman, 1982.

[8] L. Richardson, "The problem of contiguity: an appendix of statistic of deadly quarrels," General systems tearbook, vol. 61, pp. 139-187, 1961.

[9] T. Higuchi, "Approach to an irregular time series on the basis of the fractal theory," Physica D, vol. 31, pp. 277-283, 1988.

[10] L. Burlaga and L. Klein, "Fractal structure of the interplanetary magnetic field," Journal of Geophysical Research, vol. 91, no. A1, pp. 347-350, 1986.

[11] J-M. Girault, Apport des techniques de traitement du signal ò l'analyse et détection de signaux emboliques, $\mathrm{Ph}$. $\mathrm{d}$ thesis, Tours University, France, 1999. 